\title{
Optimal triple configurations of stationary shocks
}

\author{
Mihail Chernyshov ${ }^{1,}$, and Alexandr Tyapko ${ }^{2}$ \\ ${ }^{1}$ Baltic State Technical University "VOENMEH", St. Petersburg, Russia \\ ${ }^{2}$ Peter the Great St. Petersburg Polytechnic University, St. Petersburg, Russia
}

\begin{abstract}
The triple configurations of stationary shock waves with negative slope angle of the reflected shock to the velocity vector of the supersonic stream before the triple point ("inverse" triple configurations) are considered. "Inverse" configurations with maximum relations of flow parameters at the slipstream after triple point are determined. Extreme relations of the flow parameters downstream the "inverse" configurations are compared with the parameters achieved on the whole set of the tripleshock configurations that appear in supersonic streams of the inviscid perfect gas.
\end{abstract}

\section{Introduction}

Triple configurations (TCs) that form in gas flow with Mach number $M$ (Fig. 1,a-d) consist of three stationary shocks (1-3) with the common (triple) point $T$, as well as the slipstream $(\tau)$ emanating from that point $[1,2]$. Conditions of pressure equality and streamline colinearity at the sides of the slipstream $\tau$ connect the parameters of the shocks 1-3:

$$
\theta_{1}+\theta_{2}=\theta_{3}, \quad J_{1} J_{2}=J_{3}
$$

Flow deflection angle $\theta_{i}$ and Mach number $M_{i}$ downstream any shock $i$ are determined by the shock strength $J_{i}$ (relation of the static pressures after the shock and before it) and flow Mach number $M_{k}$ before that shock:

$$
\begin{gathered}
\tan \theta_{i}=\sqrt{\frac{(1+\varepsilon) M_{k}^{2}-J_{i}-\varepsilon}{J_{i}+\varepsilon}} \cdot \frac{J_{i}-1}{\gamma M_{k}^{2}-(J-1)}, \\
M_{i}=\sqrt{\left[\left(J_{i}+\varepsilon\right) M_{k}^{2}-(1-\varepsilon)\left(J_{i}^{2}-1\right)\right] /\left[J_{i}\left(1+\varepsilon J_{i}\right)\right]}
\end{gathered}
$$

(here and below $\varepsilon=(\gamma-1) /(\gamma+1)$, and $\gamma$ is the ratio of gas specific heats). The angle $\omega_{i}$ of the slope of the $i$-th shock relating to the upstream flow velocity vector also depends on its strength:

\footnotetext{
*Corresponding author: mvcher@mail.ru
} 


$$
J_{i}=(1+\varepsilon) M^{2} \sin ^{2} \omega_{i}-\varepsilon
$$
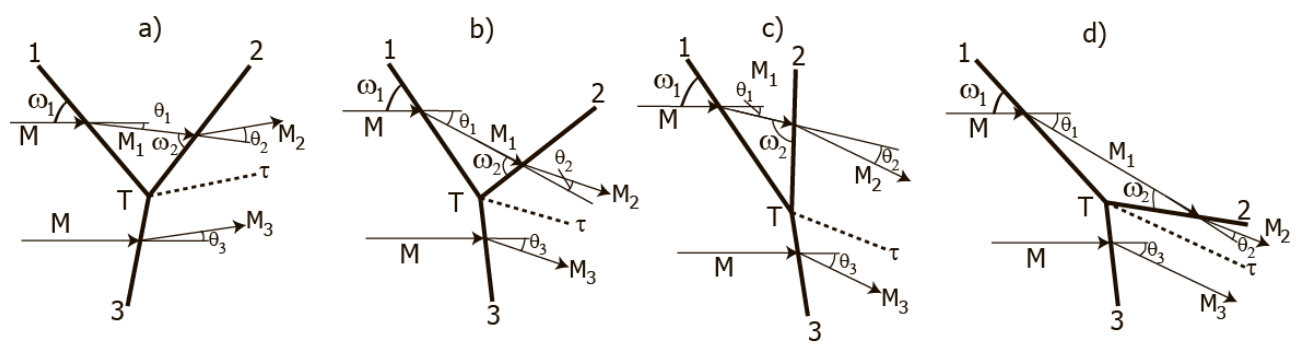

Fig. 1. Triple-shock configurations of the first (a), the second (b), and the third (c) type; "inverse" triple configurations (d)

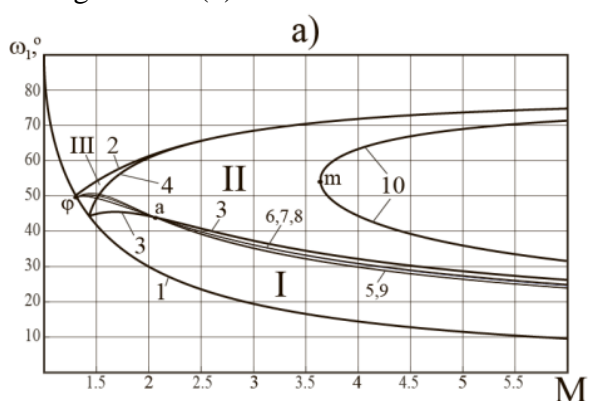

c)

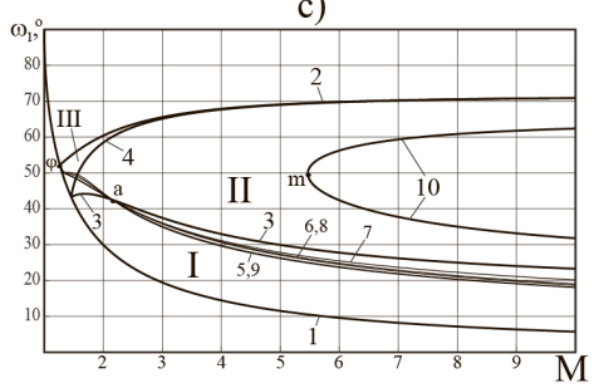

b)

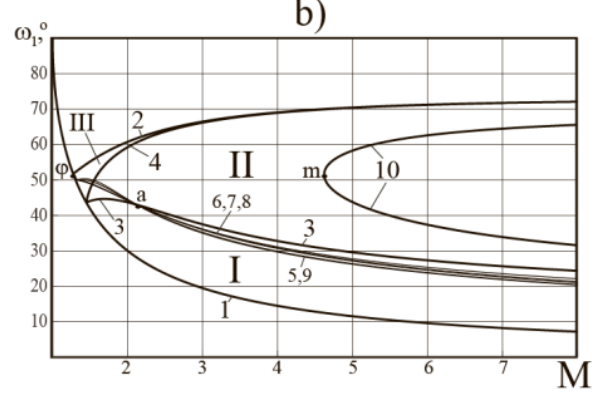

d)

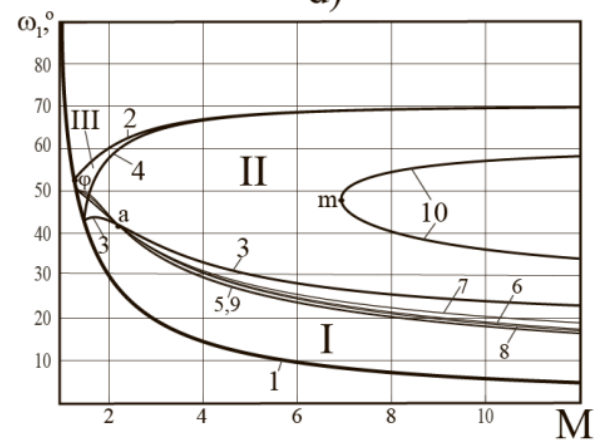

Fig. 2. Parameters of the extreme configurations that appears at $\gamma=1.1(a), \gamma=1.2(b), \gamma=1.25(c)$ and $\gamma$ $=1.3(d)$. Curves 5 correspond to configurations with extrema of the relations of densities, full pressures and other parameters outnumbered in (6); curves 6 - to the extrema of the relations of Mach numbers and dynamic pressures; curves 7-9 - to the extrema of the relations of flow velocities, flow rate functions, and flow impulses, correspondingly

Other flow parameters after the shocks 1-3 are determined by well-known relations for the perfect gas (such as Klapeyron equation of state or Hugoniot adiabat).

Depending on flow deflection directions on the shocks 1-3, we distinguish the configurations of the first (TC-1, $\theta_{1} \theta_{2}<0, \theta_{1} \theta_{3}<0$ ), the second (TC-2, $\theta_{1} \theta_{2}<0$, $\theta_{1} \theta_{3}>0$ ), and the third type (TC-3, $\theta_{1} \theta_{2}>0, \theta_{1} \theta_{3}>0$ ). As a rule, TC- 1 and TC- 3 are considered as special cases of the interaction of the counter and the overtaking shocks, correspondingly; TCs-2 are studied historically as results of irregular (Mach) shock reflection 
[3]. The most complete parametrical analysis of all types of TCs is obtained in [4-6], and the extreme TCs [5] are discovered as shock systems with the maximum relations $\left(I_{f}\right)$ of various flow parameters $(f)$ at the sides of the outgoing slipstream.

\section{Model and methods}

Triple-shock configurations are usually analyzing at the plane $\left(M, \omega_{1}\right)$ that is shown in Fig. 2,a-d. The region of the possible parameters of the incident shock 1 is situated between the curve 1 that corresponds to degeneration of that shock into the weak disturbance ( $\omega_{1}=\arcsin (1 / M)$ is Mach angle), and the curve 2 that corresponds to the degeneration of the overtaking shock 2 . The following equation $[5,7]$ determines the curve 2 :

$$
\begin{gathered}
M=\sqrt{[A(B-C)-1+\varepsilon] / \varepsilon} \\
A=\left(1+\varepsilon J_{1}\right) /\left[(1+\varepsilon)\left(J_{1}(1-3 \varepsilon)-4 \varepsilon^{2}\right)\right], B=J_{1}\left(1-2 \varepsilon-\varepsilon^{2}\right)-2 \varepsilon^{2} \\
C=2 \varepsilon \sqrt{\varepsilon\left(1+\varepsilon J_{1}\right)\left(J_{1}+\varepsilon\right)} .
\end{gathered}
$$

Curves 2 starts from the point $\varphi$ at the curve 1 that corresponds to Mach number

$$
M_{\varphi}=\sqrt{\frac{2+2 \sqrt{\varepsilon}}{1+2 \sqrt{\varepsilon}}} .
$$

Curves 3 and 4 separate the region of TC existence into zones I-III that correspond to configurations TC-1, TC-2, and TC-3. The equation of the curve 3 that separates the zones of TC-1 and TC-2 existence,

$$
\begin{aligned}
& \sum_{n=0}^{3} E_{n} J_{1}^{n}=0, \quad E_{3}=1-\varepsilon, E_{2}=-\left[\left(1+\varepsilon-\varepsilon^{2}+\varepsilon^{3}\right) M^{2}+(1-\varepsilon)\left(1-\varepsilon+\varepsilon^{2}\right)\right], \\
& E_{1}=\varepsilon\left[(1+\varepsilon) M^{2}+1-\varepsilon\right] \cdot\left[(1-\varepsilon) M^{2}-2+\varepsilon\right], \quad E_{0}=(1-\varepsilon)\left(M^{2}-1\right)\left((1+\varepsilon) M^{2}-\varepsilon\right),
\end{aligned}
$$

corresponds to well-known von Neumann criterion ("criterion of the mechanical equilibrium") of the transition of the single Mach reflection (with formation of TC-2) to the regular one.

The curve 4 that is determined by the equation

$$
\begin{gathered}
M^{4}-r M^{2}+\left(J_{1}-1\right)\left(J_{1}+2-\varepsilon\right) /(1-\varepsilon)=0 \\
r=\left(J_{1}-1\right)\left(J_{1}+2-\varepsilon\right) /\left(J_{1}+\varepsilon\right)+\left(J_{1}+\varepsilon\right) /(1+\varepsilon)+\left(1+\varepsilon J_{1}\right)^{2} /\left[(1-\varepsilon)\left(J_{1}+\varepsilon\right)^{2}\right]
\end{gathered}
$$

is a border of TC-2 and TC-3 existence zones. It corresponds to the transition from ordinary Mach reflection to "von Neumann reflection" [3] that realizes very seldom in steady gas flows. So, the triple configurations of the second type realize usually at irregular shock reflection. 
The "inverse" TCs (ITCs) with negative slope angle of the reflected shock 2 (Fig. 1,d) to the undisturbed flow velocity vector upstream the triple point were discovered experimentally [8] and studied theoretically [9-11] at Mach reflection research in multiatomic gases. The following condition determines the ITCs:

$$
\omega_{2} \leq\left|\theta_{1}\right|
$$

As it was shown in [9], ITCs belong to the second (TC-2) type and appear usually at Mach reflection in streams of gas with small ratios of the specific heats $(\gamma<1.392)$ and large Mach number ( $M>3.064$ at $\gamma \rightarrow 1$ ). Significant differences of stagnation and dynamic pressures, Mach numbers, flow velocities and other parameters of the streams divided by the slipstream after ITC can lead to flow instability and flow blocking [12], though flow with ITC remains stable sometimes [13]. Moreover, the analysis of significant differences of flow parameters after triple points can assist to solve the stability problem of the engines of the various types [14-16].

The extreme relations $\left(I_{f}\right.$ ) of various flow parameters downstream ITCs are determined in this study analytically and numerically. They are compared with the maximum values that can be obtained at the whole set of the voluntary triple-shock configurations.

\section{Results and discussion}

\subsection{Extreme triple-shock configurations of the voluntary type}

The relations of full (stagnation) pressures $\left(f=p_{0}\right)$, temperatures $(f=T)$, sound speeds ( $f=a)$, acoustic impedances $(f=z=\rho a)$ and some other flow parameters downstream TC are in a power-law dependence on the analogical relation $I_{\rho}=\rho_{2} / \rho_{3}$ of the perfect gas densities after the shocks 2 and 3 [5]:

$$
\begin{gathered}
I_{p_{0}}=p_{02} / p_{03}=I_{\rho}^{(1+\varepsilon) / 2 \varepsilon}, I_{T}=T_{2} / T_{3}=1 / I_{\rho}, \\
I_{a}=a_{2} / a_{3}=1 / \sqrt{I_{\rho}}, \quad I_{z}=z_{2} / z_{3}=\sqrt{I_{\rho}} .
\end{gathered}
$$

The relations of velocities $(f=v)$, flow rate functions $(f=q=\rho v)$, dynamic pressures $\left(f=d=\rho v^{2} / 2\right)$, and flow impulses $\left(f=b=p+\rho v^{2}\right)$ are, above it, the functions of Mach numbers downstream the TC (in particular, they depend on its relation $\left.I_{M}=M_{2} / M_{3}\right)$ :

$$
\begin{gathered}
I_{v}=v_{2} / v_{3}=I_{M} / \sqrt{I_{\rho}}, I_{q}=\rho_{2} v_{2} / \rho_{3} v_{3}=I_{M} \sqrt{I_{\rho}}, I_{d}=\rho_{2} v_{2}^{2} / \rho_{3} v_{3}^{2}=I_{M}^{2}, \\
I_{b}=\left(p_{2}+\rho_{2} v_{2}^{2}\right) /\left(p_{3}+\rho_{3} v_{3}^{2}\right)=\left(1+\gamma M_{2}^{2}\right) /\left(1+\gamma M_{3}^{2}\right), \text { wherein } p_{2}=p_{3} .
\end{gathered}
$$

Depending on value of $J_{1}$ (or $\sigma_{1}$ ), the relations $I_{f}$ of flow parameters after TC vary from the unity (at the curves 1 and 2, Fig. 2,a-d) to some maximum value that exists at any fixed flow Mach number $M$ before TC. The slope angles $\omega_{1}$ of the incident shocks that form the triple configurations with the maximum values of the functions (6-7) correspond to the curves 5-9 in Fig. 2. That "extreme" TCs belongs to the first type at moderate and large 
Mach numbers $\left(M>M_{a}, M_{a}=\sqrt{4-3 \varepsilon+\varepsilon^{2}} /(1-\varepsilon)\right)$. Corresponding "optimal" slope angles of the shock 1 satisfy the inequality:

$$
\omega_{1}^{(\rho)}<\omega_{1}^{(b)}<\omega_{1}^{(q)}<\omega_{1}^{(M)}<\omega_{1}^{(v)}
$$

It is evident from the equations (6-7) that the TCs, that is extreme for all flow parameters outnumbered in (6), coincide: $\omega_{1}^{(\rho)}=\omega_{1}^{\left(p_{0}\right)}=\omega_{1}^{(T)}=\omega_{1}^{(a)}=\omega_{1}^{(z)} ; \quad$ moreover, $\omega_{1}^{(M)}=\omega_{1}^{(d)}$.

All curves 5-9 that correspond to the extreme TCs come from the point $\varphi$ and cross again in point $a$ at the curve 3. At $M_{\varphi} \leq M<M_{a}$, the extreme TCs belong to the second type or the third one, and their determining angles $\omega_{1}$ are in correlation that is inverse to (8).

The relations (6-7) of the flow parameters after the extreme TCs that can be achieved at various Mach numbers correspond to the curves 1-10 in Fig. 3,a-b.
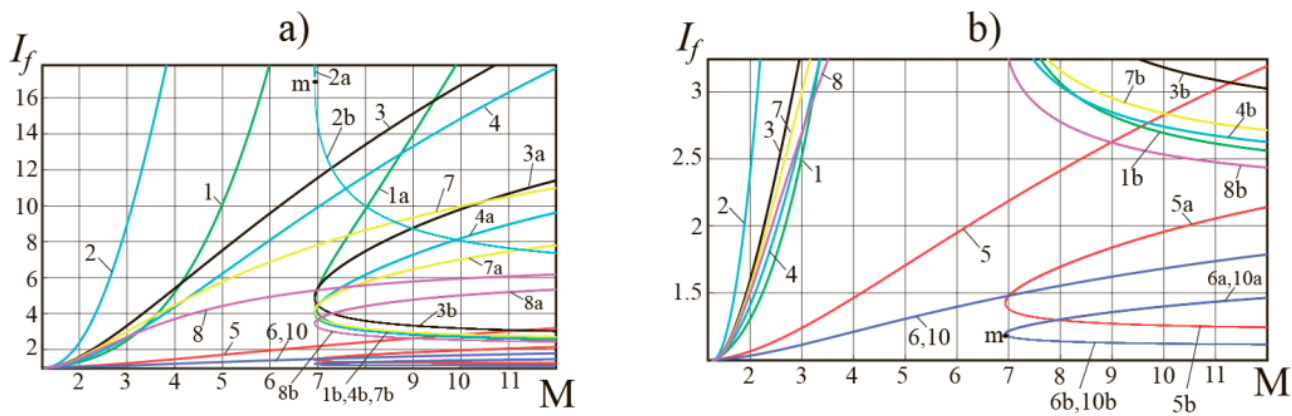

Fig. 3. Relations of flow parameters after the extreme (curves 1-10) and inverse (1a-10a, 1b-10b) triple configurations at $\gamma=1.3$ and various Mach numbers. Curves 1 show the extreme relations of stagnation pressures, 2 -dynamic pressures, 3 - flow rate functions, 4 - flow impulses, 5 - densities, 6 - acoustic impedances, 7 - Mach numbers, 8 - velocities, 9 - temperatures, 10 - sound speed (inverse relation). Curves 1a-10a, 1b-10b correspond to the relations of those parameters at the lower and the higher borders of ITC existence region

In hypersonic limit $(M \rightarrow \infty)$, the extreme relations of various flow parameters depend on the ratio of the specific heats only:

$$
\begin{gathered}
I_{\rho} \rightarrow 1 / \varepsilon=7.667, I_{p_{0}} \rightarrow \varepsilon^{-(1+\varepsilon) / 2 \varepsilon}=6812, I_{T}=\varepsilon=0.1304, I_{a}=\sqrt{\varepsilon}=0.3612, \\
I_{z}=1 / \sqrt{\varepsilon}=2.769, I_{M}=\sqrt{(K+N) / 2 \varepsilon^{3}}=18.51, I_{v}=\sqrt{(K+N) / 2 \varepsilon^{2}}=6.686, \\
I_{q}=I_{b}=\sqrt{(K+N) / 2 \varepsilon^{4}}=51.26, I_{d}=(K+N) / 2 \varepsilon^{3}=342.7, K=(1-\varepsilon)^{2}+2 \varepsilon^{3}, \\
N=(1-\varepsilon) \sqrt{(1+\varepsilon)\left(1-3 \varepsilon+4 \varepsilon^{2}\right)},
\end{gathered}
$$

(see curves 1-10 in Fig. 4). Since the "inverse" TCs do not appear at $\gamma=1.4$ (traditionally used for computational examples), here and below the computational results are obtained at $\gamma=1.3$.

\subsection{Relations of flow parameters downstream the "inverse" triple configurations}

The condition (5) excludes all TCs, that are not "inverse", out of the consideration. The region of ITC existence, that is determined by (5), is bordered by the curve 10 in Fig. 2,a-d. As it is 
seen from the Fig. 2, the ITCs exist, starting from some minimal Mach numbers: $M_{m}=3.639$ at $\gamma=1.1 ; M_{m}=4.621$ at $\gamma=1.2 ; M_{m}=5.466$ at $\gamma=1.25$; $M_{m}=6.933$ at $\gamma=1.3$.

According to [5], the voluntary extreme TCs belong to the first type at such large Mach numbers, but the ITCs [9] belong to the second type everywhere. Consequently, the ITCs cannot be extreme in the whole set of voluntary triple configurations, and flow parameters downstream them are always smaller than after the extreme voluntary configurations. The relations of flow parameters after the ITCs increase monotonously from the higher branch of the curve 10 to its lower branch. Those relations are presented in Fig. 3,a-b: by the curves 1a$10 \mathrm{a}$ - for the lower border of ITC existence region, by the curves $1 \mathrm{~b}-10 \mathrm{~b}$ - for the higher one.

a)

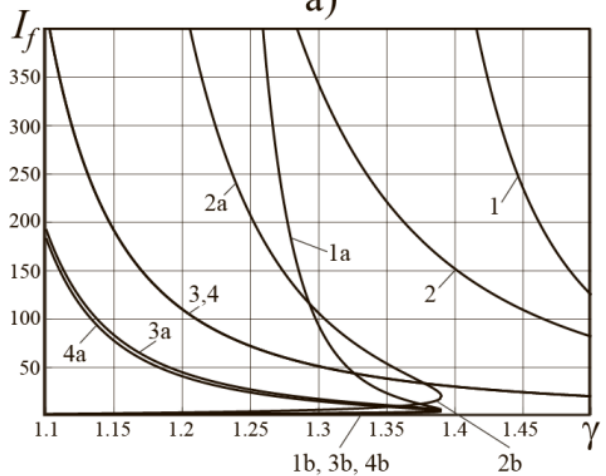

b)

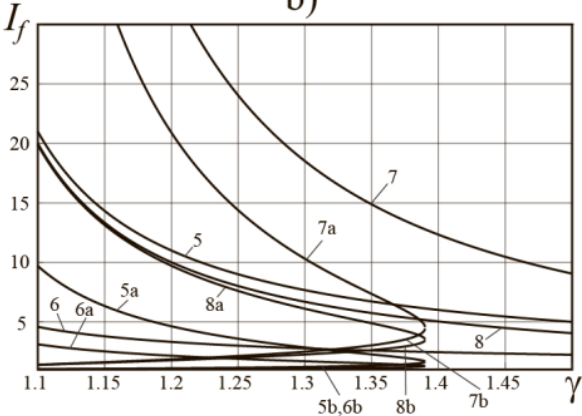

c)

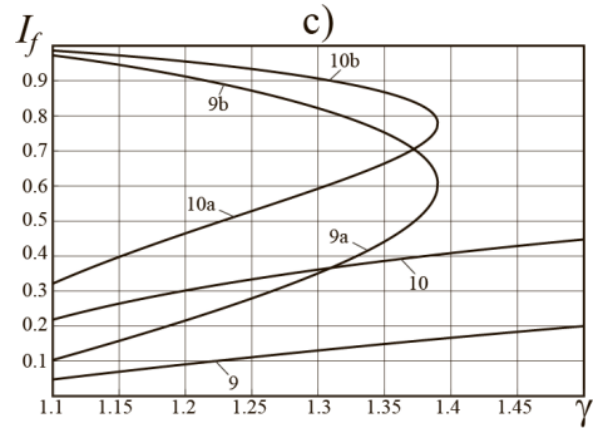

Fig. 4. Limit relations of flow parameters at large Mach numbers after the voluntary extreme (curves 1-10) and "inverse" (1a-10a, 1b-10b) triple configurations. Curves 1 present the extreme relations of stagnation pressures, 2 - dynamic pressures, 3 - flow rate functions, 4 - flow impulses, 5 - densities, 6 - acoustic impedances, 7 - Mach numbers, 8 - velocities, 9 - temperatures, 10 - sound speed (inverse relation). Curves $1 \mathrm{a}-10 \mathrm{a}, 1 \mathrm{~b}-10 \mathrm{~b}$ correspond to the largest and the smallest relations of those parameters downstream the "inverse" TCs

The limit relations of flow parameters after the ITCs obtaining at large Mach numbers can be described as it follows (see also curves 1a-10a at Fig. 4):

$$
\begin{gathered}
I_{\rho}=\frac{1+\varepsilon-C}{\varepsilon(2 \gamma-C)}=2.850, I_{p_{0}}=\left[\frac{1+\varepsilon-C}{\varepsilon(2 \gamma-C)}\right]^{\frac{1+\varepsilon}{2 \varepsilon}}=93.50, I_{T}=\frac{\varepsilon(2 \gamma-C)}{1+\varepsilon-C}=0.3509, \\
I_{a}=\sqrt{\frac{\varepsilon(2 \gamma-C)}{1+\varepsilon-C}}=0.5924, I_{z}=\sqrt{\frac{1+\varepsilon-C}{\varepsilon(2 \gamma-C)}}=1.688, I_{M}=\sqrt{P /[(2 \gamma-C) R]}=10.30,
\end{gathered}
$$




$$
\begin{gathered}
I_{v}=\sqrt{\varepsilon P /[(1+\varepsilon-C) R]}=6.103, I_{q}=(2 \gamma-C)^{-1} \cdot \sqrt{(1+\varepsilon-C) P /(\varepsilon R)}=17.39, \\
I_{d}=P /[(2 \gamma-C) R]=106.2, I_{b}=\frac{(\gamma-C)\left[\gamma(1+\varepsilon)-\gamma(2-3 \varepsilon) C+(1-\varepsilon) C^{2}\right]}{(2 \gamma-C)\left[\gamma^{2} \varepsilon-\gamma(1-2 \varepsilon) C+(1-\varepsilon) C^{2}\right]}=15.13 .
\end{gathered}
$$

Here $P=\gamma(1+\varepsilon)-\gamma\left(3-2 \varepsilon-4 \varepsilon^{2}\right) C+(1+\varepsilon)(3-4 \varepsilon) C^{2}-(1-\varepsilon)^{2} C^{3}$, $R=\gamma \varepsilon-(1+\varepsilon)(1-2 \varepsilon) C+(1-\varepsilon)^{2} C^{2}$, and $C \in(0 ; 1)$ are limit (asymptotic) values of $\sin ^{2} \sigma_{1}$ at $M \rightarrow \infty$ on the higher and the lower borders of ITC existence region. The equation

$$
\begin{gathered}
(1-\varepsilon)^{3} C^{5}-\left(1-\varepsilon^{2}\right)(4-5 \varepsilon) C^{4}+(1+\varepsilon)\left(6-2 \varepsilon-7 \varepsilon^{2}\right) C^{3}-2 \gamma^{2}\left(2-2 \varepsilon-\varepsilon^{2}\right) C^{2}+ \\
+\gamma^{3}\left(1+\varepsilon-\varepsilon^{2}-3 \varepsilon^{3}\right) C-\varepsilon \gamma^{4}\left(1-\varepsilon+2 \varepsilon^{2}\right)=0
\end{gathered}
$$

determines them. The largest relations of flow parameters downstream ITCs determined in (10) can be achieved choosing the smaller root of (11) from the range $C \in(0 ; 1)$. The larger root of (11) corresponds to the higher branch of ITC existence region. It determines the "inverse" TCs with the smallest relations of flow parameters downstream them (see curves $1 b-10 b$ in Fig. 4).

\section{Conclusion}

The extreme relations of flow parameters downstream the "inverse" TCs correspond to the lower border of their existence region. Their values concede to the corresponding maximum values after the voluntary TSs; but they are very large nevertheless, and it can influence sufficiently on the stability of supersonic flows with triple configurations in various facilities. "Inverse" configurations do not exist at the larger values of gas specific heats' ratio ( $\gamma \geq 1.392$ ).

This work was financially supported by the Ministry of Education and Science of the Russian Federation (agreement No. 14.577.21.0277, a unique identifier of applied scientific research RFMEFI57717X0277).

\section{References}

1. L.D. Landau, E.M. Lifshitz, Course of Theoretical Physics: Vol. 6. Fluid Mechanics. Oxford: Butterworth-Heinemann, 539 (1987)

2. Adrianov A.L., Starykh A.L., Uskov V.N. Interference of Stationary Gasodynamic Discontinuities. Novosibirsk: Nauka, 180 (1995)

3. G. Ben-Dor, Shock Wave Reflection Phenomena. Berlin - Heidelberg - NewYork: Springer, 342 (2007)

4. L.F. Henderson, Aeronautical Quarterly 15, 181-197 (1964)

5. V. N. Uskov, M. V. Chernyshov, Journal of Applied Mechanics and Technical Physics 47, 492-504 (2006)

6. H. Hekiri,G. Emanuel, Physics of Fluids 27 (5), 056102 (2015)

7. A. V. Omel'chenko, V. N. Uskov, Fluid Dynamics 31, 597-603 (1996)

8. L.G. Gvozdeva,S.A. Gavrenkov, Technical Physics Letters 38(4), 372-374 (2012)

9. M. V. Sil'nikov, M. V. Chernyshov, L. G. Gvozdeva, Technical Physics 61, 1633-1637 (2016) 
10. S.A. Gavrenkov, L.G. Gvozdeva, Technical Physics Letters 38(6), 587-589 (2012)

11. L. G. Gvozdeva, M. V. Silnikov, S. A. Gavrenkov, Acta Astronautica 116, 36-42 (2015)

12. L.G. Gvozdeva, S.A. Gavrenkov, Progress in Flight Physics 7, 437-452 (2015)

13. G.V. Shoev, M.S. Ivanov, Thermophysics and Aeromechanics 23(3), 343-364 (2016)

14. M. V. Gravit, O. V. Nedryshkin, O. T. Ogidan, Magazine of Civil Engineering 77, 3846 (2018)

15. O. Nedryshkin, M. Gravit, K. Grabovyy, MATEC Web of Conferences 193, 03023 (2018)

16. M. V. Gravit, E. V. Golub, S. P. Antonov, Magazine of Civil Engineering 79, 86-94 (2018) 